\title{
Embedded Web Server based Interactive data acquisition and Control System
}

\author{
Miss.Pulate S.V. ${ }^{1}$, Mrs.Diggikar A.B. ${ }^{2}$ \\ ${ }^{I}$ Electronics, SPWEC,Sharanpur, Aurangabad,India, \\ ${ }^{2}$ Electronics,SPWEC,Sharanpur, Aurangabad,India
}

\begin{abstract}
Design of on-line embedded web server is a one of the difficult task of many real time data acquisition and control system applications. The global system of interconnected computer networks is called as World Wide Web which uses the standard Internet Protocol Suite (TCP/IP) to aid billion of users worldwide and enables the user to interface many real time embedded applications like data acquisition,Industrial automations and safety measures etc,. This paper tells the design and development of on-line Interactive Data Acquisition and Control System (IDACS) using ARM9 based embedded web server. It is permitted to a network, intelligent and digital distributed control system. Single chip IDACS method increses the processing speed of a system and also avoids the problem of poor real time and reliability.This system uses ARM9 Processor and RTLinux. Web server application is ported into an ARM processor using embedded ' $C$ ' language. Web pages are designed in Hyper text markup language (HTML).

Keywords - Embedded ARM9 Processor, RTLinux RTOS, Embedded web server, IDACS.
\end{abstract}

\section{INTRODUCTION}

Online Interactive Data Acquisition and Control system plays the major role in the rapid development of the fast popularization and control in the field of measurement and control systems. It has been designed with the help of many electrical, electronic and high voltage equipments; it makes the system more complicated and not reliable. This paper approaches a new system that contains inbuilt Data Acquisition and Control system (DACS) with on-line interaction. It makes the system more reliable and avoids more complication. It is the great demand in consumer applications and many industries.

This system replaces various complex cables which are used for acquisition and it uses Ethernet and ARM processor for data acquisition and digital diagnosis. There are various digital DAC systems are available for the substitution of multisite job operation. A single worker can interact with the machine and collect various data from ongoing work in a single work station. The simplest design of data acquisition system is detailed in [1], which is based on Linux Operating system [2]; it is the popular choice for many embedded real time applications and PC systems. This system process the client based on dynamic manner by server response and it maintains separate data base with DAC controller. A web server can be embedded into any appliance and connected to the Internet so the appliance can be monitored and controlled from remote places through the browser in a desktop. This brings in a need for web services being deployed on various embedded processors such as Advanced RISC Machine (ARM) in real time context.In [3] advanced traffic survey mechanism uses data collection process for post processing of vehicle's position. Signal conditioning is the major part of any data acquisition unit. High level integration architecture was discussed in [4]; it allows signals to be conditioned, simultaneously acquired according to the external clock and triggers processed and transferred data to real time servers.Signal measurement from astrophysical sources is described in [5]; where the shared memory and internet protocols are used for data handling and process from remote users. It was developed with Global Positioning System (GPS) and Environmental monitoring system. Similarly depends on industry and its location General Packet Radio Service (GPRS) also used for data transmission through on-line. But this paper doesn't use GPRS and GPS systems for data uploading into internet. It reduces the system complexity and effective for all kind of real time applications. Every real time embedded system should be run by real time operating systems. In this paper Real time Linux Operating system is ported in ARM9 processor. Generally all ARM9 processors have the portability with any kind of higher end RTOSes. Here the embedded web server application is developed and ported into ARM9 with this setup. This single ARM board has been act as data acquisition unit, control unit, embedded web server and self diagnosis. All processes are allocated with essential resources and associated with reliable scheduling algorithms and internet protocols followed by ARM processor. This miniaturized setup reduces the complexity \& size of system.

It contains an operating system, web pages to run the application and a large memory space for server functionality. When the configured IP address is entered in the web browser, the predesigned HTML web pages gets displayed through which we can remotely monitor and control the sensor and device status respectively. The heart of communication is TCP/IP protocol. Network communication is performed by the IEEE 802.3 
Ethernet standard. ARM processor is chosen because ARM has high data processing capability. It also has multi parameter acquisition and multi level monitoring and networking. It can reduce operation pressure on data reduction and ensures real-time monitoring of system performance. On the other hand, our proposed web server requires only a very low d.c. supply of $3.3 \mathrm{~V}$ for its operation. This system ensures portability and high reliability. When RTOS is incorporated into this system, more devices could be controlled and monitored. The multi tasking capability and fast response time along with added advantage of easy deployment makes this system capable for a wide variety of applications. The system designed here is an example of embedded technology integrated with network technology where communication and processing technology also meets.

\section{SYSTEM ARCHITECTURE}

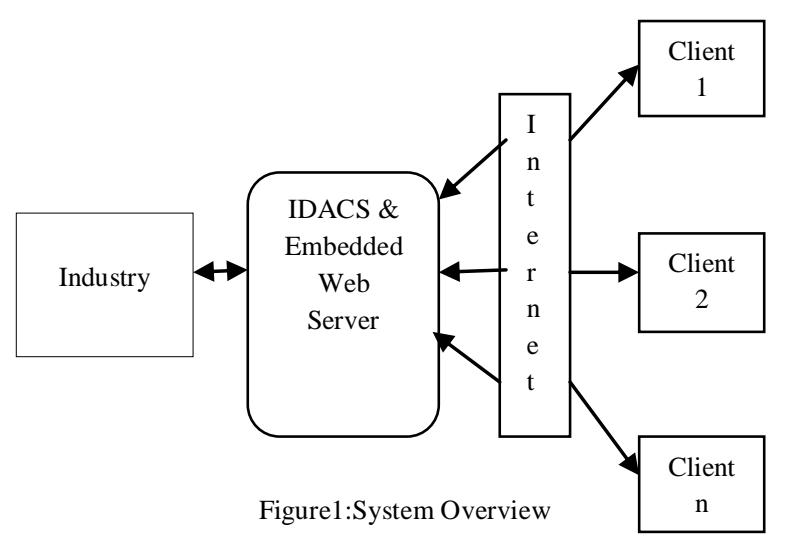

Fig. 1 shows the overview of IDAC system. Here the system consists of three different parts. First is the Embedded Web Server. Second is the user part or client and third one is the industry or sensors and appliances to be monitored. The client thus monitors various parameter status through sensors and also controls many industrial appliances with the help of the web server.Every client can access the industry directly without any interaction with additional server and modules. The client PC is connected to the Internet through a browser and then gets access to the embedded Web server. Through this way, remote login and operation are realized.IDACS shows Intelligent Data Acquisition and Control System. This system contains single ARM9 processor which is portable with Real Time Linux RTOS. ARM processor is the heart of this work. It handles two modes at same time, DAC and Web server.During DAC mode Processor can measure signals which are coming from various external sources and applications. And it can control the industry machineries by the control instruction sent by client via embedded web server. During signal measurements Analog to digital converter is very important, because almost every external source is giving analog signal only. While converting these analog to digital processor has to handle asynchronous interrupts.

This system uses RTLinux so it can handle many interrupts in an efficient manner because RTLinux has preemptive kernel with required privilege levels. Similarly during web server mode processor will handle client request and response to the particular client by sending web pages, client can interact the industry by giving instruction in web page on its own web browser. This setup can be suitable for inter communication with other nodes via Ethernet and higher end ports. Ethernet programming and execution is very easy and adaptable with various applications. Embedded web pages are designed by HTML language. Fig 1. Shows the proposed concept of Data Acquisition and Control Systems (DACS) with embedded web server. It contains RTOS portable ARM processor. The real time operating system manages all the tasks such as measuring signals, conversion of signals, data base up-dation, sending HTML pages and connecting/communicating with new users etc.The RTOS manages all the required tasks in parallel and in small amounts of time. Web based management user interfaces using embedded web server have many advantages: ubiquity, user-friendly, low-development cost and high maintainability.

\section{SYSTEM DESIGN}

Hardware design, Software design and Porting are the entire important steps in whole system design.

\section{A Hardware design of the system \\ 1) IDACS Design:}

IDACS design is the major part in hardware. ARM9 processor is a centre core of this system. The general hardware structure of the IDACS is shown in Fig 2. The online intelligent data acquisition and control system based on embedded ARM platform has high universality, each acquisition and control device equipped with 24-way acquisition/control channels and isolated from each other.Each I/O channel can select a variety of 
electrical and nonelectrical signals like current, voltage, resistance etc.,Digital acquisition are done by special ADC. The measured data are stored in external memory in which the memory is act as a data base during web server mode. The ARM9 Processor directly supports the Ethernet service and RS485 communication. Hence the data has been stored and controlled by some other PCs or network via RS485 \& Ethernet. ARM processor has internal $\mathrm{I} 2 \mathrm{C}$ module. So it has the ability to communicate with any other peripherals.

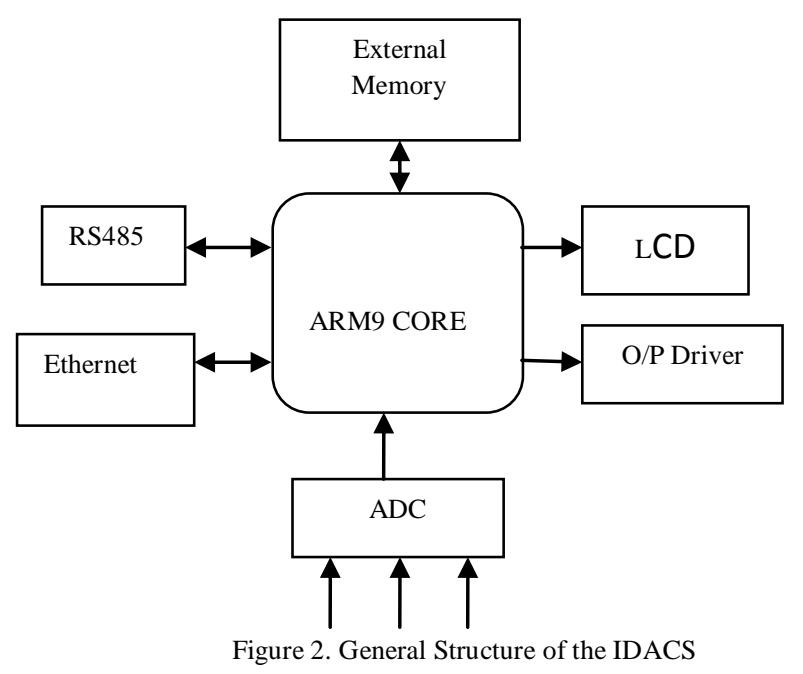

I2C is the wired communication protocol to communicate with other processor or peripherals through two wired link. This system has $128 * 64$ LCD to display the information and measured parameters which makes the debugging and modification of the parameter easy. The Analog to digital interfacing module is independent with the embedded system, which is beneficial to the system maintenance and upgrade. As the embedded Ethernet interface makes the remote data exchange between the applications become very easy.The Linux sockets are used to communicate between ARM server and webpage's form clients. The communication between ARM platform and client board is by RS232 protocol. A PIC microcontroller act as the CPU of the client board.

\section{2) Analog to Digital Converter:}

Fig 2. uses 16bit ADC chip AD7715. This is digital chip having I2C module internally. It has the ability to transfer the converted digital data to ARM processor. It needs only five lines, which are DOUT - Data output, DRDY - Data ready, DIN - Data Input, CS - Chip select and SCLK - system Clock. Converted digital data will be sending out by DOUT pin of the chip. This ADC chip is driven by $2.4576 \mathrm{MHz}$ crystal. It contains separate Reference signals Ref+ and Ref- and separate Analog input channels AIN+ and AIN- . During communication with ARM processor this ADC chip should be synchronized with the processor's clock.

\section{3) RS485 Communication:}

RS-485 is a telecommunications standard for binary serial communications between devices. It is the protocol or specifications that need to be followed to allow devices that implement this standard to communicate with each other.This protocol is an updated version of the original serial protocol known as RS-232. While the original RS-232 standard allowed for the connection of two devices through a serial link, RS-485 allows for serial connections between more than 2 devices on a networked system.

\section{B. Software design of the system \\ 1) Real Time Linux:}

RTCore is a POSIX 1003.13 PE51 type real-time kernel,something that looks like a multithreaded POSIX process with its own internal scheduler. RTCore can run a secondary operating system as a thread, using a small virtual machine to keep the secondary system from disabling interrupts. This is a peculiar model: a UNIX process with a UNIX operating system as a thread, but it provides a useful avenue to modularity. RTLinux is RTCore with Linux as the secondary kernel. RTCore with BSD UNIX as the secondary kernel. Real-time applications run as real-time threads and signal handlers either within the address space of RTCore or within the address spaces of processes belonging to the secondary kernel. Real-time threads are scheduled by the RTCore scheduler without reference to the process scheduler in the secondary operating system. The secondary operating system is the idle thread for the realtime system. The virtual machine virtualizes the interrupt controller so the secondary kernel can preserve internal synchronization without interfering with real-time 
processing. Performance is adequate to allow standard PC and single board computers to replace DSPs in many applications.

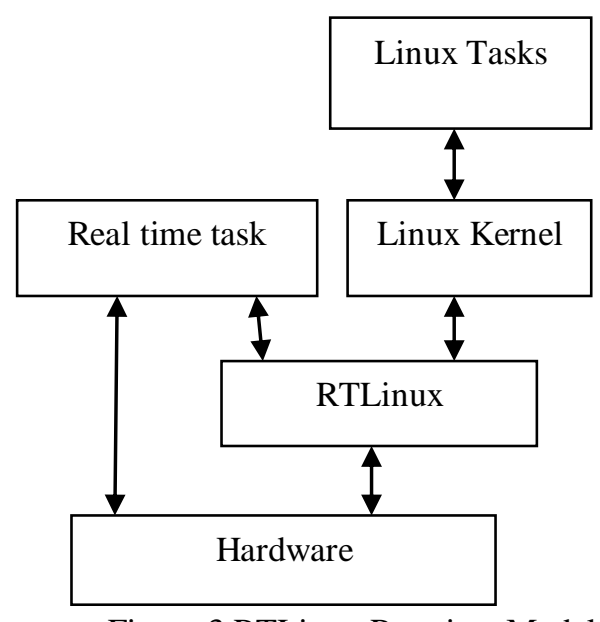

Figure 3.RTLinux Run time Model

Unlike Linux, RTLinux provides hard real-time capability. It has a hybrid kernel architecture with a small realtime kernel coexists with the Linux kernel running as the lowest priority task. This combination allows RTLinux to Provide highly optimized, time-shared services in parallel with the real-time, predictable, and low-latency execution.Besides this unique feature, RTLinux is freely available to the public. As more development tools are geared towards RTLinux, it will become a dominant player in the embedded market. RTLinux is a typical dualkernel, one is Linux kernel, which provides various features of general purpose OS, other one is RTLinux kernel, which support hard real time capability.

\section{PROPOSED System}

General web server requires more resources and huge amount of memories. This system can only measure the remote signals and it cannot be used to control the process.

Limited processing capacity and the problem of poor real time and reliability of DAS system has been overcome by the substitution of embedded ARM processor for single chip method to realize interactive data acquisition and control (IDACS). This IDACS system can able to measure the remote signals and can control the remote devices through reliable protocols and communication network. This system uses RTLinux Multitasking operating system to measure and control the whole process. And the embedded web server mode requires less resource usage, high reliability, security, controllability and portability.

\section{CONCLUSIONS}

With the rapid development of the field of industrial process control and the wide range of applications of network, intelligence, digital distributed control System, it is necessary to make a higher demand of the data accuracy and reliability of the control system. This embedded ARM system can adapt to the strict requirements of the data acquisition and control system such as the function, reliability, cost, size, power consumption, and remote access and so on. This system operated by DACS mode to acquire the signals and control the devices remotely. Embedded web server mode is used to share the data with clients in online.Both modes are efficiently carried out by real time multi tasking operating system (RTLinux). This system can be widely applied to electric power, petroleum, chemical, metallurgy, steel, transportation, Electronic \& Electrical industries, Automobiles and so on.

\section{REFERENCES}

[1] K.JackerandJ.Mckinney, "TkDAS- A data acquisition system using RTLinux, COMEDI, and Tcl/Tk," inProc. Third Real Time Linux Workshop, 2001. [Online].Available: The Real TimeLinuxFoundation:http://www.realtimelinuxfoundation.org/events/rtlws2001/papers.html

[2] E.Siever, A.Weber, S.Figgins, and R.Love, CA:O’Reilly, "Linux in a Nutshell,”,2005.

[3] J. E. Marca, C. R. Rindt, M. Mcnally, and S. T. Doherty, "A GPS enhanced in-vehicle extensible data collection unit," Inst. Transp.Studies, Univ. California, Irvine, CA, Uci-Its-As-Wp-00-9, 2000.

[4] Y. C.Wu, J. R. Luo, and J. Z. Shan, "Development of the central timing system on the EAST Tokamak," IEEE Trans. Nuclear science, Vol. 30, No. 9, pp. 789-792, 2007.

[5] F. Acernese, P. Amico, M. Alshourbagy, F. Antonucci, S. Aoudia, P.Astone, S. Avino, D. Babusci, G. Ballardin,’Data Acquisition System of the Virgo Gravitational Waves Interferometric Detector,"IEEE Trans. Nuclear science, Vol. 55, No. 1, pp.225-232, February 2008

[6] D. Vijendra Babu, P. Subramanian and N. Ravi Kanan, "Microblaze and Uclinux Based Data Acquisition on Spartan 3E" Proc. Of International Conference on VLSI Design and Embedded Systems,pp1-4, 2008. 
[7] S. B. Silverstein, J. Rosenqvist, and C. Bohm, "A simple Linux-based platform for rapid prototyping of experimental control systems,"IEEE Trans. Nucl. Sci., vol. 53, no. 3, pp. 927-929, Jun. 2006.

[8] Peng D.G, Zhang H., Yang L., etc, "Design and Realization of Modbus Protocol Based on Embedded Linux System". The 2008 International Conference on Embedded Software and Systems Symposia. July 29-31, 2008, Chengdu, Sichuan, China, pp:275-280.

[9] Soumya Sunny P1, Roopa .M2; "Data Acquisition and Control System Using Embedded Web Server" International Journal of Engineering Trends and Technology- Volume3Issue3- 2012.

[10] Alen Rajan, Aby K. Thomas; "ARM Based Embedded Web Server for Industrial Applications" International Conference on Computing and Control Engineering (ICCCE 2012), 12 \& 13 April, 2012

[11] Linux Kernel API ,http:www.kernel . org/doc/htmldocs/kernelapi/index.html

[12] RTLinux - http://www.rtlinux.org

[13] www.opensource.org

[14] www.embeddedarm.com 